Olivar, vol. 19, $\mathrm{n}^{\circ}$ 29, e054, mayo-octubre 2019. ISSN 1852-4478

Universidad Nacional de La Plata.

Facultad de Humanidades y Ciencias de la Educación.

Centro de Estudios de Teoría y Crítica Literaria

\title{
Centro/periferia: la cuestión de la soberanía lingüística en La Carreta y en La prensa cegetista (Argentina, 1940-1941; 1952-1953)
}

\author{
Pulgarcito Magazine (1904-1907): The formation of a children's audience in Argentina
}

\author{
Mara Glozman \\ CONICET / Facultad de Filosofía y Letras - Universidade Buenos Aires, Argentina \\ maraglozman@gmail.com
}

\section{ReSUMEN:}

El artículo analiza dos series de textos aparecidos en dos publicaciones periódicas argentinas: La Carreta y La Prensa. El análisis observa, en particular, dos momentos relevantes en materia de debates sobre la lengua: 1940-1941 en el caso de La Carreta y 1952-1953 en el caso de La Prensa. Se trata de escritos breves que reinscriben, en dos coyunturas diferentes, elementos de una formación que está presente en los debates argentinos desde el segundo tercio del siglo XIX: el lazo político-lingüístico e institucional con España. El trabajo enmarca el análisis de las dos series delimitadas en una problematización más general: la relación entre lengua y soberanía en los discursos producidos en Argentina. Con el fin de historizar y comprender los procesos de formación de esta cuestión constitutiva, se presenta una caracterización de dos formaciones diferentes: la reivindicación del legado hispánico como elemento de soberanía frente al proyecto panamericanista de los Estados Unidos; la vindicación de la soberanía lingüística nacional frente al gobierno español de la lengua. Es esta segunda formación, como muestra el artículo, la que produce efectos en los escritos de La Carreta (1940-1941) y de La Prensa (1952-1953).

Palabras clave: Soberanía lingüística, Argentina, Formaciones discursivas Anti-hispanismo Primer peronismo

\begin{abstract}
:
The article analyzes two series of texts that appeared in two Argentinean periodicals: La Carreta and La Prensa. The analysis observes, in particular, two relevant moments in terms of debates on the language: 1940-1941 in the case of La Carreta and 1952-1953 in the case of La Prensa. These are brief writings that reinsert, in two different conjunctures, elements of a formation that is present in the Argentine debates since the second third of the 19th century: the political-linguistic and institutional bond with Spain. The work frames the analysis of the two series delimited in a more general problematization: the relationship between language and sovereignty in the discourses produced in Argentina. In order to historicize and understand the processes of formation of this constitutive issue, a characterization of two different formations is presented: the vindication of the Hispanic legacy as an element of sovereignty against the Pan-American project of the United States; the vindication of national linguistic sovereignty against the Spanish government of the language. As the article shows, it is this second formation that produces effects in the texts of La Carreta (1940-1941) and La Prensa (1952-1953).
\end{abstract}

KeYwords: Language sovereignty Argentine, Discursive formation, Anti-hispanism, First Peronism

\section{INTRODUCCIÓN}

Sujetos a los procesos de formación propios de las materialidades discursivas, los discursos sobre la lengua están habitados por expresiones, articulaciones, relaciones de sentido y formas de decir que -transformados al calor de los distintos momentos históricos- retornan de manera recurrente. En el caso de los discursos sobre la lengua producidos en Argentina, la cuestión de la soberanía nacionales uno de esos elementos. Se hace presente, bajo modalidades diversas, en un vasto conjunto de textos elaborados entre el segundo tercio del siglo XIX y la actualidad. Atraviesa un universo discursivo heterogéneo; constituye, en la historia argentina, uno de los aspectos fundantes de los debates sobre la lengua.

Atender a los funcionamientos de esta cuestión, a sus coyunturas de (re)emergencia, a las modalidades que la configuran, en suma, al modo en que se significa la contradicción entre soberanía/emancipación y dependencia/dominaciónpermite construir un archivo, entendido como principio de organización de materiales. Desde un punto de vista descriptivo, la contradicción soberanía-dependencia es un sí una relación, 
esto es, es una función que articula elementos: el objeto a defender o a propulsar -por ejemplo la nación, la emancipación nacional, la lengua, el legado hispánico-, por un lado, y el sujeto que amenaza o subordina, esto es, el elemento respecto del cual opera la (in)dependencia, efectiva, afectiva, imaginada -por ejemplo, España, la Real Academia Española, la Unión Panamericana, la cultura anglosajona, Estados Unidos, el capitalismo internacional, las lenguas extranjeras-. Desde un punto de vista procedimental, la contradicción soberanía-dependencifunciona - retomando la propuesta de Guilhaumou, Maldidier y Robin (2016) - como dispositivo de archivo: permite escandir los discursos, distinguiendo formaciones diferenciadas según cuáles sean y cómo se presenten los elementos que participan.

En este marco, el presente trabajo analiza dos publicaciones periódicas: la revista político-cultural La Carreta (en adelante, LC), editada durante décadas por el Círculo Leales y Pampeanos de Avellaneda, y el suplemento cultural del diario La Prensa (en adelante, LP), en el lapso en que la empresa estuvo bajo la conducción de la Confederación General del Trabajo, luego de ser expropiada por el Estado en 1951. El trabajo no aborda las publicaciones periódicas como unidad en sí; en cambio, realiza un recorte de ciertas zonas, de ciertas piezas de los materiales, seleccionadas en función de dos criterios: la articulación lenguasoberanía y la estabilización de un momento determinado por ciertas condiciones de producción.

En el caso de LC, nos detenemos específicamente en una serie de notas publicadas en 1940 y 1941; se trata de un momento relevante porque confluyen varias cuestiones que generan efectos en los discursos sobre la lengua: la adopción de la hispanidad como política del Estado español tras el triunfo franquista, el desarrollo de la industria editorial de habla hispana con sede en Buenos Aires, la creciente difusión mediática de obras de divulgación gramatical prescriptiva orientadas a un público amplio, la circulación de discursos que procuran definir y, algunos de ellos, reivindicar formas del lenguaje consideradas populares. En esta coyuntura, LC publica un conjunto significativo de ensayos breves que tienen como blanco las principales figuras de los años '40: Avelino Herrero Mayor y su gramática normativa; Amado Alonso y sus publicaciones sobre lengua, libros y filología hispánica; el Consejo de la Hispanidad español, entre otras.

En el caso de LP, nos detenemos puntualmente en dos notas publicadas durante el segundo gobierno peronista, momento de reemergencia de los debates sobre la lengua, en particular de las posiciones que reivindican la soberanía del Estado nacional en materia idiomática, en detrimento de los lineamientos político-lingüísticos de las instituciones normativas existentes, en general, y de la Real Academia Española, en particular. En efecto, 1952-1955 es un período de intensa producción de escritos sobre la lengua y de intervenciones polémicas desde diversas inscripciones, tanto en la línea de las políticas antihispanistas gubernamentales expresadas en el SegundoPlan Quinquenal(1952) y en sus instrumentos de difusión como de posiciones que ensayaban una defensa del legado hispánico ante aquello que se percibía como un ataque político a la lengua española y a las academias tradicionales.

Las dos series, así organizadas, participan a nuestro entender de una misma formación discursiva, esto es, de una de las matrices en tensión que proporcionan sentidos y articulaciones para hablar acerca de cómo la lengua se vincula con el problema de la soberanía nacional. Es de este modo que encaramos el análisis: siguiendo la línea teórica pecheutiana (Pêcheux, 2012; Pêcheux, 2016) y la relectura analítica de las secuencias que conforman, las series son entendidas como elementos de una formación que las excede y constituye; los sentidos que allí se formulan participan, al mismo tiempo, de un dominio de actualidad y de dominios de memoria, que sobredeterminan aquello que puede y debe ser dicho y que silencian u "olvidan" otras formas de anudar lengua y soberanía.

Dadas estas condiciones, la exposición se dispone en dos instancias. La sección II presenta una caracterización general de los discursos sobre la lengua en Argentina, atendiendo al dispositivo de archivo establecido: la relación soberanía/dependenciaEl objetivo de este rodeo es dislocar la evidencia de homogeneidad y unicidad en las interpretaciones del sintagma "soberanía lingüística" e inscribir las series recortadas en una de las posibles formaciones. Las secciones siguientes presentan una caracterización de los 
materiales (Sección III) y un análisis de las dos series que organizan el trabajo: LC 1940-1941 (Sección IV) y el suplemento cultural de LP 1952-1953 (Sección V).

\section{Lengua y Soberanía en Argentina}

La relación entre lengua y soberanía aparece en la historia argentina significada de modos diversos, que varían según el momento de producción de los discursos, su inscripción institucional, su dimensión polémica, las filiaciones de las posiciones enunciativas que operan como marca de autoría, la inscripción de esta articulación en formaciones que sobredeterminan los elementos de la escena enunciativa. Sería posible, pues, identificar diversas modalidades de esta articulación según el aspecto sobre el cual el análisis coloque la mirada.

En posibilidad es pensar esta cuestión en relación con los procesos de inmigración extranjera (véase, por ejemplo, Di Tullio, 2003). Un caso específico en esta línea es el análisis de Bertoni (2001), que muestra posiciones que, ante la masiva inmigración italiana en el contexto de fines del siglo xix, temían por un posible reclamo de soberanía sobre el Río de la Plata. También podría pensarse esta problemática en relación con la apelación a la lengua francesa, que aparece en varios escritos como sustento para reivindicar la autonomía de una "lengua argentina" o un "idioma nacional" emancipado del español; ejemplo de ello son algunas publicaciones de la denominada generación de 1837 (véase Varela, 1999) y uno de los ensayos que ha generado mayor controversia en su contexto de aparición: Idioma nacional delosargentinos(1900), de Lucien Abeille.

En otra dirección, es importante mencionar la existencia de discursos que señalan la filiación "amerindia" o "indígena” como rasgo diferencial americano. Tal es el caso del emblemático ensayo Eurindia (1924) -también otras producciones de Ricardo Rojas (véase Martínez Gramuglia, 2006; Bentivegna, 2011; Bentivegna, 2018; Lagos, 2014): la apelación a las culturas indígenas funciona como elemento de un entramado identitario heterogéneo que incluye y destaca el legado hispánico. El gesto de incorporar las culturas y lenguas indígenas a la matriz nacional bien puede inscribirse en un movimiento más amplio, que tuvo en las primeras décadas del siglo XX expresiones relevantes en América Latina (véanse Funes, 2006; García-Romeu, 2012). Esta conjunción entre lenguas indígenas e hispanismo se hace presente, a lo largo del siglo XX, en diversos documentos de política pública, entre ellos el Plan de Gobierno1947-1951. Las lenguas indígenas aparecen también en diversos documentos de la década de 1940 que legitiman la política panamericanista impulsada desde Washington.

Si bien los modos en que se anudan lengua y soberanía son múltiples, el trabajo de archivo realizado hasta el momento nos ha permitido distinguir dos grandes matrices, dos formaciones dominantes. Ambas involucran necesariamente un posicionamiento ante una relación que se caracteriza como subordinación, actual o potencial, como relación de dependencia a transformar mediante la construcción de soberanía o como potencial injerencia o dominación externa que amenazaría la soberanía ya constituida; la variación de los elementos que componen esa relación estructural es lo que permite distinguir las dos formaciones.

La primera se caracteriza por la vindicación de la soberanía lingüística frente a España, o frente a las políticas que reverberan el colonialismo español en los países americanos; el momento fundacional se suele ubicar en la década de 1830, en los vastamente analizados escritos de Juan María Gutiérrez, Domingo F. Sarmiento, Juan Baustista Alberdi, Esteban Echeverría. Se trata de una formación cuyos elementos retornan de manera incesante en cada nueva emergencia que habilita discusiones sobre la lengua en los foros de lo público (véase Glozman y Lauria, 2012; Alfón, 2013; Arnoux, 2008; Ennis, 2008). Los sintagmas “soberanía lingüística” o "soberanía idiomática" suelen interpretarse en el sentido de esta primera formación.

La segunda formación puede ser caracterizada por la defensa de la lengua española y del legado hispánico/ hispanoamericano frente a las políticas de los países angloparlantes. Su momento de emergencia es posterior, hacia el último tercio del siglo XIX, momento de estructuración -en palabras de Terán (1993)- del "dispositivo hispanista" en Argentina. También es el momento de impulso del panamericanismo como un 
instrumento de la diplomacia estadounidense para legitimar la construcción de un espacio de intercambio preferencial entre Estados Unidos y los países de América Central y América del Sur (Bernecker, 2000). Profundizado el escenario beligerante en el contexto de la Guerra de Cuba, el avance de los Estados Unidos era percibido por las élites conservadoras como una amenaza a la soberanía e independencia de las repúblicas hispanoamericanas. Las alusiones de rechazo a la "doctrina Monroe" y al expansionismo estadounidense, así como la mención recurrente de las "dos Américas" como espacios geo-lingüísticos diferenciados y en tensión - la América española, la Américasajona- atraviesan, en efecto, un conjunto de textos de amplia circulación hacia fines del siglo xix y comienzos del xx; entre ellos, el conocido ensayo "El problema de la lengua en la América española" (1899), de Ernesto Quesada:

No es, pues, debida al espectáculo tristísimo que ha ofrecido la última guerra hispano-yankee, que nos sentimos inclinados a estrechar los vínculos que ligan a las repúblicas de origen español con la madre patria. Nada más natural que así se piense en el sud del continente americano; como lo es que en el norte del mismo se entonen loores a la pujanza y a los éxitos de la raza anglo-sajona en cualquier parte del mundo. (...) No se crea que es exagerada esta importancia de la lengua, ni que son vanos los temores respecto de la influencia de la raza anglo-sajona en América a este respecto (...). La extensión singularísima que la política imperialista yankee ha dado en los últimos tiempos a su socorrida "doctrina de Monroe" (...) demuestra que las esferas de acción de las razas sajona y latina, en el continente americano, se encuentran en vísperas de ser violentamente antagónicas. (Quesada, 1899: 247)

Esta articulación también puede observarse en diversos documentos de los primeros años de gobierno peronista. En este sentido, la dirección dominantemente "hispanista" que tomaron los discursos de políticas públicas entre 1946 y 1949 puede ser (no solo pero también) comprendida desde esta perspectiva. En la coyuntura de posguerra, entre el nuevo impulso del panamericanismo impulsado desde Washington y las políticas hispanistas de la España franquista, el entonces presidente Perón pronuncia una alocución pública en el marco del homenaje a Cervantes realizado el 12 de octubre de 1947 en la Academia Argentina de Letras a instancias del "Día de la Raza":

Porque la difusión de la leyenda negra, que ha pulverizado la crítica histórica seria y desapasionada, interesaba doblemente a los aprovechados detractores. Por una parte, les servía para echar un baldón a la cultura heredada por la comunidad de los pueblos hermanos que constituimos Hispanoamérica. Por la otra procuraba fomentar así, en nosotros, una inferioridad espiritual propicia a sus fines imperialistas, cuyos asalariados y encumbradísimos voceros repetían, por encargo, el ominoso estribillo cuya remunerada difusión corría por cuenta de los llamados órganos de información nacional. Este estribillo ha sido el de nuestra incapacidad para manejar nuestra economía e intereses, y la conveniencia de que nos dirigieran administradores de otra cultura y de otra raza. Doble agravio se nos infería; aparte de ser una mentira, era una indignidad y una ofensa a nuestro decoro de pueblos soberanos y libres (...).

Son hombres y mujeres de esa raza los que en heroica comunión rechazan, en 1806, al extranjero invasor (...); es la que se derramó generosamente cuantas veces fue necesario para defender la soberanía y la dignidad del país; es la misma que moviera al pueblo a reaccionar sin jactancia pero con irreductible firmeza cuando cualquiera osó inmiscuirse en asuntos que no le incumbían y que correspondía solamente a la nación resolverlos; de esa raza es el pueblo que lanzó su anatema a quienes no fueron celosos custodios de su soberanía, y con razón, porque sabe, y la verdad lo asiste, que cuando un Estado no es dueño de sus actos, de sus decisiones, de su futuro y de su destino, la vida no vale la pena de ser allí vivida. ("La fortaleza de nuestra raigambre hispánica”, en Boletines de la Academia Argentina de Letras XVI, 1947: 489-480)

Así, desde el punto de vista de las formaciones que organizan los discursos sobre la lengua -tal como los hemos delimitado más arriba - en estas formulaciones hispanismo y rechazo a la cultura anglosajona resultan elementos estructuralmente vinculados.

Ahora bien, en el caso de la primera formación, la vindicación de emancipación frente a la historicidad de los procesos de conquista y colonización que marcaron, de manera constitutiva, la memoria de los países americanos está significada por otro elemento: el tiempo y su relación con el desarrollo. España, la antigua Metrópoli, es vista, pues, como factor que atrasa. Como señala Arnoux (2008), la imagen de una sociedad y de un conjunto de instituciones -la monarquía, la Real Academia Española, las formas de sus gramáticas y diccionarios estatales- retardatarias e incapaces de comprender los procesos de transformación de la modernidad aparece configurada desde el segundo tercio del siglo XIX; basta con releer algunas zonas de los 
escritos sarmientinos Memoria (sobreortografíaamericana) leída a la Facultad de Humanidades(1843) y De la educación popular(1849).

Lo retardatario será también un elemento de sentido que, ya en la década de 1920 y comienzos de los años '30, volverá a ser vinculado con las gramáticas y gramáticos españoles, con la filología hispánica y las instituciones a ellas asociadas:

El catalán Sr. Montoliu y el americano-ibero don Américo Castro, favorecidos por la autoproclame de unos eurindiosde la Universidad de Buenos Aires, vinieron a esta nuestra generosa tierra como adelantados y correjidoresllamados para fundar y dirijir un Instituto de Filolojia en la Facultad de Filosofía y Letras, con el atrevido plan de sujerir la irremediable obligación de entregarnos incondicionalmente al "habla española", si queremos conceptuarnos cultoshidalgos de este virreinato del rio de la plata, antes negrero y ahora lengual. (Rossi, 1929: 5)

Señor Monner Sans: si le hiciéramos caso a la gramática, tendrían que haberla respetado nuestros tatarabuelos, y en progresión retrogresiva, llegaríamos a la conclusión de que, de haber respetado al idioma aquellos antepasados, nosotros, hombres de la radio y la ametralladora, hablaríamos todavía el idioma de las cavernas (Arlt, 1958 [1930]: 156).

Es esta segunda formación que genera efectos de sentido en los fragmentos recortados para la conformación de las series, con reformulaciones y nuevas paráfrasis: en la década de 1940 y a comienzos de 1950 la cuestión de lo popular participa de los discursos que abogan por una soberanía lingüística frente a España.

\section{Materiales y Recorte: MOMENTOS, POSICIONES, Figuras}

Las series seleccionadas presentan otros rasgos en común: el carácter cultural de las publicaciones en las que participan y cierto rasgo vinculado con las condiciones materiales de edición de las publicaciones, que tienen efectos en el lugar desde el cual se interviene públicamente en la cuestión. En ambos casos la proclama de una posición que vindica la soberanía lingüística e institucional frente a las políticas españolas y a la defensa de la "lengua común" se realiza desde un lugar que material y simbólicamente podríamos caracterizar, en principio de manera intuitiva, como periférico y que tensa las representaciones en torno de las prácticas culturales en sus contextos de circulación.

LC era el órgano del Círculo de Leales y Pampeanos del partido de Avellaneda, una de las principales asociaciones tradicionalistas de la provincia de Buenos Aires; fundado con ese nombre en 1932, celebraba reuniones de payadores, jineteadas y otras formas de preservación de las prácticas ritualizadas asociadas a la cultura gauchesca y al costumbrismo campero, incluyendo actividades artísticas y una escuela de danzas tradicionales (véase Casas, 2016). LC está, en efecto, sobrecargada de referencias a la figura del gaucho; su mismo título, "La Carreta", es indicio de la inscripción proyectada y del efecto identitario tradicionalista que envuelve la publicación.

De la extensa edición de esta publicación, hemos observado - tal como señalamos en la Introducción- un momento específico: el período 1940-1941. En primer lugar, se trata de un momento en el que procesos distintos confluyen en una reemergencia hegemónica de la defensa de la "lengua española común" y de la “inscripción hispánica”: la institucionalización del franquismo y la consecuente expansión de las políticas de "Hispanidad" (véase Sepúlveda, 2005); la radicación y el desarrollo en Buenos Aires de editoriales vinculadas a emigrados y exiliados españoles - por ejemplo Losada, de la que participaba Amado Alonso (véase, por ejemplo, Ferrero de Sahab et al., 1993) -; la ampliación del público lector y la proliferación de publicaciones sobre gramática y léxico que apuntaban, con tono cordial y prescriptivo, a las crecientes capas medias urbanas (véase Glozman, 2017); la publicación de nuevos volúmenes e intervenciones en la prensa de las principales figuras vinculadas a la filología hispánica en Buenos Aires, Amado Alonso y Américo Castro (véase Toscano y García, 2013). También es posible observar una circulación de "enunciados hispanistas" en diversas instancias de la producción documental estatal. Para la Academia Argentina de Letras, creada poco después de proclamada la Segunda República Española, es el momento de estrechar lazos con una 
Real Academia que adscribía, al menos en sus comunicaciones oficiales, de manera explícita a las políticas del franquismo. Los Boletinesde la Academia Argentina de Letras publicados en 1940-1941 son una cabal expresión del estrechamiento de tales lazos político-académicos en los años inmediatamente posteriores a la derrota republicana de 1939. Al menos una parte de los materiales producidos en el Ministerio de Justicia e Instrucción Pública compartieron tal orientación, bajo el mando ministerial de Guillermo Rothe, quien había ocupado el mismo cargo en el gobierno dictatorial de José F. Uriburu e impulsado la creación de la Academia Argentina de Letras en 1931.

En segundo lugar, con una fuerza mayor, la selección se sustenta en el modo en que aparecen aspectos de estas dimensiones, figuras y procesos dentro de LC. Es notable, en ese breve lapso, la profusión de notas en torno del "hispanismo" y el "idioma nacional", que marcan desde los títulos la voluntad de intervenir en el debate público: "Rubén Darío y la Hispanidad”, “¿Hispanofobia?”, "Sueño de 'Imperio Idiomático”, "Américo Castro 'Corregidor' de Lengua'...", "Amado Alonso y su idiomilla de coleccionista”, "Señor gramatiquero", "El señor Herrero y el valor de las citas", "Sarmiento en una cita del señor Herrero", "Por nuestro idioma nacional”, “Lo castellano' en el castellano", "La pureza en el lenguaje”, "Estancamiento de la lengua", "El purismo idiomático", "Rebeldías antiacadémicas", "La campaña del idioma".

La relevancia de exponer esta serie no reside solamente en dar a conocer las ideas de una publicación que resulta subalterna en términos de producción del conocimiento y en las esferas político-culturales; esta serie de notas no predica solamente acerca de las posiciones de LC. Los breves y numerosos ensayos de LC sobre la lengua muestran cuáles fueron y cómo se organizaban los saberes metalingüísticos en la Argentina de principios de la década de 1940. Esta serie de notas señala con eficacia nombres, tesis, problemas, ámbitos de las diversas voces y lugares enunciativos que se entrecruzan en los debates públicos.

Para este trabajo consideramos de LC, de manera prioritaria, los textos firmados por Luis C. Pinto, figura sin dudas menos conocida que Vicente Rossi, el otro autor cuyas notas, tomadas de los Folletoslenguaraces, también eran publicadas en la revista. Los títulos citados corresponden, todos, a la producción de Pinto. Este nombre interesa también por su itinerario autoral posterior, que lo llevará a escribir folletos y materiales elogiosos de las políticas sobre la lengua enunciadas por el gobierno peronista en el SegundoPlan Quinquenal (1952).

La segunda serie, cuantitativamente menor, se inscribe en otras condiciones de producción. Se trata de dos artículos publicados en el Suplementocultural del diario La Prensa durante el segundo gobierno peronista: "El problema de nuestro lenguaje" (1952), de Arturo Cambours Ocampo, e "Independencia de nuestro idioma nacional" (1953), de Amílcar Medina Verna. La indicación de los años de publicación es fundamental; significa que las notas que consideramos son posteriores a la expropiación, en 1951, de LP, momento a partir del cual queda bajo la gestión de la CGT (Confederación General del Trabajo).

El suplemento cultural, no obstante, mantenía una cierta independencia respecto de los lineamientos sindicales e, incluso, respecto de los modos en que se delimitaba en otros ámbitos -instituciones educativas formales, por ejemplo- la inscripción al peronismo a comienzos de los años ' 50 . En efecto, bajo la dirección de César Tiempo, el Suplementocultural albergó notas y figuras política y estéticamente heterogéneas (véase Panella y Rein, 2013). A diferencia de la caracterización presentada en torno de LC, en el caso del suplemento cultural de LP resulta problemático afirmar que, en tanto instancia de enunciación e intervención en los debates públicos, se tratara de un espacio periférico en la composición del campo cultural e intelectual de aquella coyuntura. Principalmente porque tal afirmación supondría contar con un consenso generalizado en torno de la estructuración de las esferas culturales durante el primer peronismo (véanse, entre otros, Korn, 2017; Leonardi, 2017). Empero, es posible pensar que una publicación cultural producida bajo el sello de la CGT supone, al menos, horadar ciertos imaginarios dominantes respecto de cuáles son los centros legitimados para la producción de prácticas culturales y para la elaboración de publicaciones que predican acerca de las actividades culturales. Es en este sentido que habilitamos la interrogación por la relación centroperiferia para caracterizar las condiciones editoriales de los textos seleccionados. 
Hay, en la coyuntura 1952-1953, también una diferencia respecto de LC 1940-1941 en lo que concierne a los campos, instituciones y figuras. Se trata de un momento singular en la historia de los discursos metalingüísticos. El gobierno peronista desde principio de los años '50 tensó las relaciones con los centros tradicionalmente legitimados para la producción de saberes prescriptivos y epistémicos sobre la lengua: en 1952 las actividades de la Academia Argentina de Letras se encontraban suspendidas y había disminuido la centralidad de las publicaciones producidas por el Instituto e Filología Hispánica de la Universidad de Buenos Aires. 1952-1953 es un momento de intensificación, expuesta, explícita, de discusiones sobre el papel y el derecho del Estado nacional a legislar en materia lingüística, sobre la injerencia de la Real Academia Española en la producción de diccionarios, sobre, en suma, un haz de elementos que reverberan expresiones, relaciones de sentido y articulaciones provistas, en perspectiva histórica, por la formación "antihispanista" ya caracterizada. 1952-1953 es un momento en el que las instancias de gobierno del Estado -especialmente, pero no solo, a través de las formulaciones del Segundo Plan Quinquenal (1952) y sus instrumentos de difusión- asumen tal posición como propia, cuestionan, con un gesto eminentemente político, las formas de producción del saber institucionalizadas en Argentina desde los años '20 y '30 (véase Glozman, 2015). Los dos artículos sobre la lengua publicados en el LP durante el segundo gobierno peronista participan de aquel dominio de actualidad.

\section{LA CARReta 1940-1941: combates DESDE los MÁRgenes}

Como hemos mencionado, en LC confluyen, en tiempo y espacio así como en la orientación de sus escritos, dos figuras que promueven, con su nombre y firma, una intensa batalla de ideas contra el hispanismo en sus diferentes vertientes. En este sentido, Pinto -más que Rossi- se dedica a polemizar; su trabajo no es tanto sobre las formas de la lengua como sobre las posiciones en juego:

Cuando hayamos adquirido una cultura, leyendo otros idiomas que no sea el 'castillano', cuando nos sobrepongamos al prejuicio idiomático madrepatriero; cuando tengamos plena conciencia de nuestra nacionalidad; cuando barramos con el lastre del colonialismo que aún perdura en el país; cuando seamos bastante fuertes para reírnos a mandíbula batiente de las pretensiones de los refugiados íberos y otros asimilados, que quieren imponernos la 'unidad del idioma', que ellos no han podido realizar en su patria; cuando, en fin, lleguemos a poseer la salud de un pueblo libre, culto y fuerte, entonces tendremos una literatura propia y habremos impuesto el idioma que habla hoy nuestro pueblo, y que desconocen sus literatos "dirigidos" y sus filólogos importados... (Pinto, 1940a: 6)

Si hacemos una delimitación tomando como criterio el dispositivo autoral, es preciso señalar un dato: para una periodización de archivo de los textos de Rossi, estas son las últimas instancias; en el archivo Pinto, en cambio, la seguidilla de encendidos ensayos breves de LC representa el punto inicial de una secuencia de publicaciones que se extenderá hasta la década de 1960 (véase Glozman, 2018). Subordinando la delimitación de la unidad de autor, y vistos los textos en conjunto, las formulaciones muestran continuidades y recurrencias que aproximan de manera sustantiva los escritos de Rossi producidos desde los años '20 y las notas de LC, incluidas las firmadas por Pinto. Es notable, pues, la persistencia de un núcleo casi invariante que se manifiesta, no obstante, en condiciones muy diferentes.

En particular, el texto "Por nuestro idioma nacional" recupera ya desde el título la forma de nombrar la lengua que aparece en Cosas de negrołRossi, 1926):

El lector deducirá el objeto del chiste de los derrotistas de nuestro idioma nacional en próspero avance, que los tiene profundamente preocupados, pues no hay argumento posible contra esa obra incontenible de la Evolución, con todas sus causales, circunstancias y consecuencias (Rossi, 1926: 400)

Otro elemento recurrente que marca la proximidad entre los textos de Rossi de los años '20 y las notas de LC es la expresión "vasallaje idiomático”, que significa el debate sobre la lengua como dimensión de una 
batalla, aún no acabada, entre el mantenimiento de las relaciones coloniales -con España- y la obtención de la independencia lingüísticąque, en esta matriz, también es cultural y política

No comprendemos como hay quienes hablan de argentinidad en el idioma, y colman sus aspiraciones 'nacionalistas' en una tímida solicitud a las autoridades 'competentes' a fin de 'legalizar' algunas particularidades fonéticas, (el 'yeyeo' y el 'seseo', por ejemplo). Hablar, en nuestro país, de 'matices', 'particularidades', o 'pasatiempos' lexicográficos, es hacer de todo menos obra nacionalista. Existe un problema, sí; pero es muy vasto y fundamental. No se puede hablar de reformas sin antes reconocer ciertos hechos, bien patentes por poco que se profundice la investigación: que el idioma de los argentinos, aun con sedimento castellano, evoluciona constantemente hacia la formación fonética, lexicográfica, sintáctica - de un idioma diferente, más dulce, más rico, más culto...; que no debe gastarse pólvora en chimangos, debatiéndose por reformas parciales que, de conseguirse, no alterarían absolutamente en nada nuestro vasallaje idiomático a las directivas exteriores (Pinto, 1941a: $3)$.

Si desear ardientemente la independencia de nuestro país, y de toda indoamérica, de aquel vasallaje es ser hispanófobo no podemos negar que lo somos decididamente, por muy remoto que esté nuestro sueño de la realidad (Pinto, 1940b: 19)

"Vasallaje idiomático" forma parte de un conjunto de expresiones que retoman de manera literal los Folletos lenguaraces de fines de los años '20, vocabulario que -como se observa en las formulaciones transcriptas en III- insiste sobre la continuidad entre el sistema político de la conquista y colonización ibérica -puntualmente en la región rioplatense-, con sus cargos y funciones -"adelantado", "corregidor"- y aquella actualidad de las políticas lingüísticas:

Varios siglos de predominio español han demostrado qué pueden dar de sí los íberos "conquistadores" y los descendientes "colonizadores"... Picarismo e incultura; caciquismo, politiquería y frailerío son la preciada herencia de los "fijosdalgo" madrepatrieros... (Pinto, 1940b: 19)

El señor Castro, castellanista profesional, ha sido "introducido" en el país como "adelantado" de la academia madridense y "corregidor" de nuestras "peculiaridades" lingüísticas. (...) ¿Con qué derecho pretenden ejercer sobre nosotros tutoría? ¿Con qué cultura adquirida venir a enseñarnos? ¿Con cuáles obras imponer su autoridad? (Pinto, 1941c: 3-4)

Los escritos de Pinto encaran una "campaña de revisión idiomática" (1941a: 3) que abre un frente de batalla con cada una de las principales figuras-tendencias de su dominio de actualidad. Uno de los blancos lo constituyen las instituciones vinculadas a las políticas españolas de la "Hispanidad"; la nota "Sueño de "Imperio Idiomático" ubica en posición de antagonista a las tendencias falangistas de la dictadura española; es el escrito que introduce el problema del imperialismo de manera más precisa:

Hemos leído recientemente que las pretensiones de 'recuperación' imperialistas de la falange íbera se basan, especialmente, en el 'dominio' y la 'extensión' de la lengua 'española'. (...) Ignoran los falangines que los imperios no se conquistan, ni se han conquistado nunca, con la 'lengua'... (...) ¿No deberían reconocer los españoles que quienes le retienen el Peñón tendrían derecho a dominar casi todo el mundo basados en la misma causa que ellos invocan? (...) La verdad es que el inglés es un dominio efectivo, y el español, en cambio, es la pesadilla de un imperialismo insomne en la noche oscura de su tragedia íntima... (Pinto, 1941b: 3)

No obstante, en una coyuntura internacional sumamente compleja, atravesada por los efectos de la guerra civil española y por la segunda guerra mundial, los escritos de LC sobre la lengua buscan una "tercera" posición en uno y otro conflicto:

Los que no nos casamos con ningún gobierno imperialista o tendencia internacionalista sabemos que la libertad que ansiamos del tutelaje peninsulero no nos lo proporcionará ni facilitará ninguna de las naciones en lucha, cualquiera de ellas, que triunfase en la guerra.

Solo las revoluciones internas de los pueblos indoamericanos podrán sacudirse el yugo de cuatro siglos de ignominia... Tampoco admitimos excepciones con ciertas facciones que, siendo españolas, se dicen en Argentina antiimperialistas o anti-católicas; aquí todos los españoles forman el frente unido de los "recuperadores" y de los "amos" y nos consideran despectivamente a los indoamericanos que, no obstante, somos la mayoría. (Pinto, 1940b: 19) 
Otros escritos están destinados, directamente, a polemizar con las dos figuras principales de la filología hispánica en Argentina: Amado Alonso y Américo Castro. En lo que concierne a Américo Castro, es conocido el acontecimiento que surgió en torno de la publicación de su libro La peculiaridad rioplatensey su sentidohistórico(1941) (véanse, por ejemplo, Degiovanni y Toscano y García, 2010; Bentivegna, 2011). La tesis de Castro sobre los "rasgos de desorden" y "de desquiciamiento" del idioma a orillas del Río de la Plata (1941: 9-10) generó numerosas respuestas de escritores e intelectuales argentinos, provenientes de diversas esferas y ámbitos de las prácticas culturales, entre ellos el ensayo borgeano "Las alarmas del Dr. Américo Castro". El efecto de tal escenario fue una confluencia, en la reacción contra el volumen de Castro, de sectores ideológica, cultural y socialmente diferentes, incluso confrontados. En LC aparecieron dos textos referidos a esta cuestión: “Américo Castro 'Corregidor' de la Lengua...”, de Pinto, y “A los Encomenderos Idiomáticos de los Pueblos del Plata”, de Rossi. El de Rossi se destaca porque menciona de manera explícita reacciones en los dos diarios principales de la Buenos Aires de entonces:

\footnotetext{
"Nación" i "Prensa" porteños han sorprendido a sus lectores, poniendo reparos a un libro de don Américo Castro, que fustiga sin recato la arjentinidá en el idioma que hablamos. (...) "Nación” i "Prensa" porteñas estuvieron siempre de acuerdo en esa obligación a que debemos estar atados por la lengua, i mantienen en su seno "virreyes clandestinos" (que diría Jorje Luis Borjes) que la hacen cunplir con estrictés digna de mejor enpresa. Pero don Américo ha conseguido revivir en ellas una timida arjentinidá con su libro... (Rossi, 1941: 7; ortografía original)
}

Pero el blanco principal de esta diatriba contra la filología hispánica es, sin duda, otro. Del conjunto de notas y ensayos breves de LC que participan de la serie 1940-1941 el más extenso es, precisamente, el texto dedicado enteramente a Amado Alonso: “Amado Alonso y ‘su Idiomilla de Coleccionista”. Este escrito de Pinto se concentra en los desplazamientos en las posiciones de Alonso entre su volumen El problemade la lengua en América (1935) y las ideas que pone de manifiesto en una sucesión de artículos publicados en el diario La Nación, compilados luego bajo el título La Argentina y la nivelación del idioma(1943) con el sello de la Institución Cultural Española de Buenos Aires. Se trata de un escrito más complejo que los demás, en el que se combina el vocabulario propio de los textos de los '20 con una problemática "novedosa": las editoriales, el mercado editorial y los modos de sociabilidad intelectual de los exiliados republicanos en Buenos Aires. Es preciso recordar, en este sentido, que para quienes llegaron a Buenos Aires huyendo el franquismo, el mundo editorial fue, junto con la Institución Cultural Española y varios espacios universitarios, una de las principales tramas de su inserción en las redes locales (véase Schwarzstein, 2001).

La posición de Pinto es, pues, la de un rechazo visceral al desarrollo editorial que tenía lugar entonces bajo la dirección de nombres españoles, empresa que es significada en estos textos como negocio o ventajas de mercader. De esta manera, el discurso de denuncia del "vasallaje idiomático" subsumía también esta nueva dimensión de las políticas de la lengua:

\footnotetext{
Con motivo de la guerra civil, la población española tenía poco tiempo para leer y poco dinero para comprar libros. Además, la reacción del gobierno contra casi toda la intelectualidad "republicana" y sus publicaciones, hacía peligrar el negocio editorial. Así huyeron los editores y los "productores de libros" que invadieron nuestro país plantando sus comercios "papeleros" unos, y ocupando los otros los puestos que la publicidad argentina les proporcionó, espléndida como siempre con toda la resaca que impulsó a nuestras tierras las luchas intestinas peninsulares.

Aquí, los editores hispanos entraron como en terreno conquistado; utilizando a veces procedimientos poco honestos, como en el caso del escritor Tunón; con revistas de dudosa moralidad, y con su parcialidad en defensa de campañas antiargentinas repudiadas por el pueblo. (Pinto, 1941b: 3).
}

Finalmente, mencionaremos brevemente las notas que batallan contra Avelino Herrero Mayor, una de las principales voces de defensa de la "unidad de la lengua", denominada castellana en sus textos de las décadas de 1920 y 1930 y española desde inicios de la década de 1940. Para entonces, Herrero Mayor había publicado, entre otros, Artesanía y prevaricación del castellano(1931), Diccionario,lengua y estilo(1938). Colaboraba con el diario La Nación, elaborando columnas sobre normativa gramatical, y sobre las palabras convenientes y usuales, que luego serían recogidas en Condenacióny defensade la gramática (1942) y Presentey futurode 
la lengua española en América (1944). La producción de Herrero Mayor es variada pero, en líneas generales, está atravesada por una modalidad que articula las dinámicas del discurso pedagógico con un registro más informal vinculado a los medios masivos o a la ampliación del público lector. Comparados con los otros elementos de la serie, estos escritos de Pinto tienen un tono más ameno, cierto dejo de sarcasmo humorístico que combina bien con el estilo de ese género que hemos denominado "divulgación gramatical prescriptiva", en el que incluimos varias de las producciones de Herrero Mayor -algunos de sus volúmenes, notas en la prensa y, más adelante, las emisiones radiofónicas reunidas en el Diálogo argentino de la lengua. 50 lecciones para hablar y escribir correctamente(1951) - (véase Glozman, 2017). Aun así, y pese a las distancias entre la filología y el quehacer normativo de Herrero Mayor, los ensayos de LC leen ambos discursos bajo la matriz del discurso colonial:

El señor Herrero Mayor que oficia de "corregidor" de nuestra lengua desde el rotativo porteño "Nación”, apiadado de la "insuficiencia léxica" de los "gacetilleros insípidos y neologizantes" se ha echado sobre los hombros la carga atlántica de ilustrarlos, reprenderlos y aconsejarlos. (Pinto, 1940c: 5).

A Herrero Mayor pareciera, sin embargo, tomárselo Pinto menos en serio:

Pone "escolios", sermonea "disquisiciones", aplica "apostillas" y, además, para que su farmacopea lengual sea completa, no le falta su bien provisto "recetario"... (...) Diccionario en mano, como quien deshoja "la blanca margarita”, va repasando palabras diciendo: "ésta vale, ésta no vale, ésta vale, ésta no vale... según esté o no contenida en el mamotreto académico. Trabajo pesado e insustancial de acopiador de "errores", no sustancioso de lexicógrafo, el suyo da la sensación de esas composiciones escolares donde todo verborreado lugar común tiene su asiento. (Pinto, 1940c: 5)

Si en estos textos la polémica se concentra en un nombre, una publicación o un medio, en otros casos, sin detenerse en lo específico del quién es quién, caracteriza tendencias que cumplen distintas funciones en la reproducción de las relaciones de dependencia colonial:

\footnotetext{
Son tres las tendencias que se manifiestan en el ambiente.

En primer lugar están los castellanófilos que siguen pensando que los españoles son "los amos de la lengua" y que a ellos debemos ciego acatamiento. Sienten desprecio por todo lo argentino y, comprendiendo que al imponernos su lengua bozal defienden sus posiciones y negocios editoriales, tratan de mantenernos acollarados a ella.

En segundo término se encuentra la intelectualidad y publicidad argentina hispanizante que le lleva la canasta para usufructuar posiciones bien remuneradas y expectables.

Por último, tenemos ciertos intelectuales: profesores y escritores, vergonzantes de su argentinidad, que se aventuran, todo lo más, a declarar que en el habla de los argentinos sólo hay un "matiz de diferenciación" del de los castellanos... (Pinto, 1941a: 3)
}

En esta trama, la cuestión de la posición periférica o marginal respecto de las instituciones y los lugares centrales de producción de discurso legitimado -introducida en la sección III- no es solo hipótesis de lectura sobre Pinto. En la serie de LC hay, pues, una problematización de esta posición periférica; los propios textos reflexionan sobre las posibilidades enunciativas diferenciales que habilita el decir desde los márgenes: solo desde afuera del sistema caracterizado por estas tres posiciones "solidarias" puede denunciarse el afán de subordinación. En esta dirección, las voces que enuncian desde el seno de las instituciones, medios de comunicación y publicaciones centrales son presentadas como tendencias que no pueden sino ser funcionales a los intereses en cuyo beneficio ejercen el uso de la palabra:

Los de la argentinidad vergonzante y del "matiz de diferenciación” proponen sumisamente la adopción de algunas reformas: "legalización” de algunas peculiaridades fonéticas y admisión, por la Academia Española, de algunas palabritas nuestras. Con esto resuelven el problema lingüístico a su modo y pasan por innovadores...

La posición de los primeros es más fuerte porque cuentan con todos los medios para imponer sus decisiones; gobierno, curia, prensa, institutos de cultura, cátedras, academias, etc.; los mejores puestos rentados, premios y prebendas que otorgan desde arriba con los dineros del pueblo. 
Los que no comulgamos con ninguna esquina del triángulo, ni formamos parte de instituciones anuladoras de la personalidad, ni esperamos premios, ni tememos el ridículo, podemos decir lo que no pueden decir los demás. (Pinto, 1941a: 3)

Los textos de Pinto presentan, así, una caracterización de los campos en los cuales se reproducen y circulan alguna de las variantes "hispanistas" descriptas: las prácticas que responden a estas perspectivas son comprendidas como parte de la distribución de capital no solo simbólico: las publicaciones, los premios, los cargos institucionales, las notas en los principales diarios de la Argentina comportan además un interés económico preciso. En definitiva, los escritos de LC señalan algunas modalidades con las cuales el Estado y el mercado de principio de la década de 1940 garantizaban los mecanismos de reproducción del discurso que validaba públicamente "la unidad de la lengua". En este sentido, el análisis de Pinto permite trascender la indignación por las posiciones personales de sus antagonistas: solo una transformación en las instancias de reproducción de tales mecanismos generaría las condiciones de posibilidad para que la "campaña por la independencia idiomática” deviniera acción político-lingüística y “el sueño remoto”, realidad.

\section{La Prensa cegetista 1952-1953: tensiones de la integración}

Hay un conjunto significativo de formulaciones presentes en los documentos estatales de los primeros años de gobierno de Juan D. Perón (1946-1948) que pueden y suelen describirse como expresión de la adscripción peronista al "hispanismo" o a la "hispanidad" (véase, por ejemplo, Rein, 2003). Ciertamente, figuras como Herrero Mayor, la Academia Argentina de Letras, sectores de la diplomacia franquista estuvieron próximos a las instancias de gobierno en aquellos años.

Ahora bien, el modo de organizar el archivo que expusimos en las primeras secciones permite agrietar esta "unidad", que de otro modo se muestra como evidente: entre las secuencias en las cuales se pueden observar trazos de reivindicación del "legado hispánico", solo algunas responden a la dinámica estructural caracterizada en la sección II. En este sentido, en los textos de la Academia Argentina de Letras, los de Herrero Mayor, así como documentos de la "hispanidad" franquista la cuestión de la lengua no aparece asociada al problema de la soberanía ni a la amenaza de la "América sajona" sobre "los pueblos de Hispanoamérica”. Este aspecto diferencial, distintivo, resulta crucial para comprender ciertas continuidades entre dos momentos muy distintos de los discursos públicos sobre la lengua del primer peronismo: 1946-1949 y 1952-1953. Así pues, una lectura sobre los materiales de 1952-1953 que desconociera aquellas "otras" articulaciones posibles entre lengua y soberanía produciría una inmediata interpretación de que es en 1952 cuando reemerge la preocupación gubernamental por las relaciones entre lengua y soberanía. Nada más lejano: el problema de la soberanía, sus relaciones con las lenguas y las culturas está presente desde los primeros documentos estatales del peronismo. Apelando a una imagen pendular de las formaciones caracterizadas en la sección II, diremos que entre fines de los años ' 40 y comienzos de los ' 50 la orientación dominante se moviliza de una formación a la otra, en ambos casos atravesada por un modo político y soberanista de entramar los discursos sobre la lengua.

Qué explica el "pasaje" de un momento al otro es un interrogante que supone la puesta en funcionamiento de un haz de conocimientos de naturaleza diversa: aspectos de las relaciones internacionales, transformaciones en las condiciones económicas y en las relaciones de fuerza al interior de la alianza policlasista que sostenía el peronismo, reconfiguración de alianzas políticas, con sus efectos en las reformas de 1949, del organigrama ministerial, por un lado, y de la Constitución nacional, por el otro. Entre tales dimensiones la creciente tensión con importantes sectores de la Iglesia católica no es un factor menor en la reemergencia de posiciones antihispanistas. Resulta preciso, entonces, comprender este desplazamiento en la articulación lengua-soberanía como elemento de un proceso de transformación más amplio, que afectó las esferas educativas, aspectos de las políticas culturales y comunicacionales, las problemáticas vinculadas con la circulación de la información y la formación política de la ciudadanía, y también los modos de 
elaborar los instrumentos de planificación estatal. Se trata de un panorama cuya descripción excede las posibilidades del presente trabajo. No obstante, en términos amplios, podríamos decir que si los primeros años peronistas estuvieron centrados en las transformaciones económicas y en los derechos sociales y laborales "clásicos", los inicios de la década de 1950 pueden ser caracterizados como un momento de "batalla cultural". Es en ese escenario complejo que tienen lugar tanto la expropiación de LP como una nueva y creciente problematización del lenguaje, asociada a reivindicaciones caras al discurso peronista: la justicia social, la soberanía política, la independencia económica.

1952-1953 es, pues, un período singular en la historia de los debates sobre la lengua en Argentina: sin alcanzar la intensidad polémica de LC, diversos materiales producidos desde instancias estatales, entre ellos los principales instrumentos de planificación de las políticas públicas, se hacen eco de aquellas vindicaciones de soberanía lingüística frente -contra- España y las instituciones académicas tradicionales. Con ello, no estamos afirmando que LC $\mathrm{u}$ otros textos vinculados -los Folletoslenguaraces de Rossi, por caso- hayan sido efectivamente leídos, citados, traídos. Entendemos, en cambio, que las condiciones de aquella coyuntura hicieron posible, habilitaron o sobredeterminaron la reemergencia de tales enunciados.

En el escenario caracterizado aparece la palabra de Cambours Ocampo. El breve texto publicado en LP expresa una mirada sobre la cuestión que está presente en varios otros escritos del autor: una entrada a la relación entre lengua y soberanía desde la literatura y el problema de la creación literaria, más específicamente, poética. Cambours Ocampo, profesor de la materia "Introducción a la literatura" en la Universidad Nacional de La Plata, escritor, poeta, deja huellas de sus prácticas profesionales y escriturarias en el breve ensayo que publica LP:

Siempre es útil volver al encuentro de algunos temas nacionales. Este del idioma es uno de ellos. Debemos tomarlo con pinzas, pues, desgraciadamente, se presta para la tergiversación y para la réplica erudita, donde, casi siempre, aparece la conocida y tan gastada frase: falta rigor científico

La literatura es, en definitiva, la dueña del lenguaje, les guste o no les guste a los filólogos. Por su intermedio el pueblo se expresa, y el pueblo en esto, como en todos los problemas del espíritu, es soberano. Hablar formalmente de una cultura popular, no es una novedad. En lo literario, la poesía popular fundamenta gran parte de la especulación artística. (...)

La lucha del escritor hispanoamericano con su lengua, es una realidad. No se ponen de acuerdo instrumento y pensamiento. El escritor europeo tiene afiatado el mecanismo de su expresión. Nadie corre más que nadie. Aquí, en cambio, el pensamiento del escritor está desacomodado; le falta aire, es decir, palabras para cumplir su misión; y cuando las encuentra no las puede usar, pues la cultura idiomática no se lo permite. (Cambours Ocampo, 1952: 16)

La preocupación de Cambours Ocampo, en este sentido, se orienta en una primera instancia a la relación de los escritores hispanoamericanos con la lengua en el proceso creativo de escritura. La voz que trae, la voz que cita, para profundizar este problema es la de Gabriela Mistral:

El problema del escritor americano y su lenguaje es mucho más importante, más profundo. "Me conozco los defectos y los
yerros de cada una de mis mecedurasorales, y sin embargo las di y las doy ahora todas, aunque sepa que las complejas y manidas
debieron quemarse por abortadas. Una vez más yo cargo aquí, a sabiendas, con las taras del "mestizaje verbal". Y para ir
afirmando su posición y aclarando también su concepto sobre este problema de lenguaje, recuerda las burlas de una colega
española por el empeño criollo al querer forzar la poesía popular, provocando el nacimiento por voluntad... "La oía yo con
interés: un español tiene siempre derecho para hablar de los negocios del idioma que nos cedió y cuyo cabo sigue reteniendo
en la mano derecha, es decir, en la más experimentada. Pero equé quieren ellos que hagamos? Mucho de lo español ya no
sirve en este mundo de gentes, hábitos, pájaros y plantas contrastados con lo peninsular. Todavía somos su clientela en la
lengua, pero ya muchos quieren tomar la posesión del sobrehaz de la Tierra Nueva. La empresa de inventar será grotesca; la
de repetir de 'pe a pa' lo que vino en las carabelas lo es también. Algún día yo he de responder a mi colega sobre el conflicto
tremendo entre el ser fiel y el ser infiel en el coloniaje verbal". (Cambours Ocampo, 1952: 16)

Estas citas de "Ternura" (1945) de Gabriela Mistral engarzan de manera poco armoniosa con la segunda parte del artículo, en la cual Cambours Ocampo recurre a los nombres y lugares comunes de lo que podríamos denominar la "tradición del idioma nacional": critica a Alonso, trae a Abeille. El texto oscila, de allí en más, entre recoger elementos del campo literario y filosófico (por ejemplo, Vossler y Unamuno) y el 
funcionamiento de una analogía, eficaz y alineada con los fragmentos del SegundoPlan Quinquenal, entre nacionalización de la lengua y nacionalización de los trenes:

\begin{abstract}
Por otra parte, no puede extrañar a nadie que un pueblo como el nuestro, que ha incorporado a su patrimonio material los ferrocarriles ingleses, quiera incorporar a su patrimonio espiritual la lengua española, con todo el respeto que nos merecen las locomotoras fabricadas en Liverpool y la fonética inventada en Madrid (Así como hemos podido mostrar al mundo, en una reciente exposición, una locomotora totalmente construida en el país, mañana daremos, también al mundo, nuestro idioma nacional, con su fonética y su estilística). Y esto no puede ni debe molestar a nadie. Política de recuperación y soberanía es la de estos años argentinos. (Cambours Ocampo, 1952: 16)
\end{abstract}

En este texto funciona, pues, el autor como dispositivo de legitimación, como nombre y firma, pero no es posible asegurar cuál ha sido la mano corporal que intervino en la elaboración. Podemos afirmar, en cambio, que se trata de un texto que hilvana estilos y gestos de lectura heterogéneos.

La confección del Segundo Plan Quinquenal el debate parlamentario que le otorga carácter de ley nacional y la edición de numerosos y variados materiales de difusión producidos con la finalidad de exponer todos o algunos de los objetivos del nuevo plan de gobierno acontecen, precisamente, entre comienzos de 1952, cuando se publica en LP "El problema de nuestro lenguaje", y octubre de 1953, cuando aparece "Independencia de nuestro idioma nacional", de Medina Verna. El texto de Medina Verna forma parte, pues, de esa trama abigarrada y dispersa de folletos, conferencias, manuales, ensayos y afines que funcionaron en 1953-1954 como dispositivos de difusión del nuevo plan de gobierno. Su comienzo así lo señala:

Es evidente que la cultura argentina está de parabienes. El capítulo V del Segundo Plan Quinquenal, titulado "Cultura", contiene algunos fundamentos que, aplicados de inmediato, colocarán a nuestro país entre los más adelantados en esta materia, base de toda sociabilidad y progreso humano. Uno de los objetivos generales expresa que la cultura literaria será desarrollada mediante la configuración nacional de la lengua, creando a tal fin la Academia Nacional de la Lengua, que deberá preparar el Diccionario Nacional que incluirá las voces peculiares de nuestro país en sus diferentes regiones, y las usadas corrientemente en Latinoamérica. Los demás puntos del objetivo están relacionados con el fomento de la actividad editorial para la publicación de ediciones de bajo costo de obras de literatura nacional y universal, coordinando las actividades de los editores con las entidades que agrupan; con el fomento de la difusión del libro argentino en el exterior, en cuanto signifique una expresión auténtica de la cultura nacional, y con la publicación de obras de autores argentinos premiados en concursos anuales de carácter nacional. (Medina Verna, 1953: 18)

El artículo de Medina Verna trae a su actualidad aquel rasgo que habíamos señalado para esta formación: el imaginario de una España o de unas instituciones académicas atrasadas, reacias a las transformaciones, ignorantes. Este aspecto, propio de los escritos decimonónicos antihispanistas, que reaparece luego -según vimos en la sección II- en la década de 1920, marca también las producciones de 1952-1953:

Ya no dependerán nuestras palabras de la institución matriz del idioma. En el breve prólogo que aparece en la edición del
diccionario de la Real Academia Española correspondiente al año 1947, puede leerse una advertencia que es sumamente
conveniente transcribir, por cuanto se percibe en ella el valor primordial que se ha otorgado a nuestras sugestiones, siempre
respetadas, pero que quedan, por otra parte, bien disimuladas y postergadas con estas expresiones: "Se ha decidido reimprimir
el cuerpo de la obra según se halla en la edición $16^{2}$, añadiéndole, en un copioso suplemento, las novedades más importantes
que se deducen de las recientes aportaciones y de los estudios ya efectuados, y dejando para más adelante las enmiendas
introducidas en muchos artículos. Entonces -y aquí queríamos venir a parar- habrá llegado la ocasión de utilizar, entre
otras observaciones recibidas, las muy copiosas e interesantes que se ha dignado formular la Academia Argentina de Letras".
Preciso es hacer notar que no podemos vivir pendientes de la rancia academia en esto de nuestro idioma nacional. (Medina
Verna, 1953: 18)

El artículo firmado por Medina Verna muestra otro tipo de heterogeneidades enunciativas -en el sentido de Authier-Revuz (1984, 1998)-, bien características de aquella coyuntura: no es contra las instituciones que se dirige la enunciación; aquello que se construye es una posición de demarcación. Esto es: demarcarse de las instituciones académicas, sin horadar la legitimidad del aparato institucional en sí; el primer peronismo gubernamental fue, en este sentido, claramente institucionalista. Hay, así, una tensión contenida, que se 
expresa en vacilaciones y efectos de retome de lo ya dicho o en la presencia de elementos que tenderían a orientaciones argumentativas opuestas:

\footnotetext{
España no verá con malos ojos esta notable iniciativa del Segundo Plan Quinquenal del general Perón. Al contrario, aplaudirá el paso dado por su vigorosa hija de la América del Sur, ya que nuestros escritores han otorgado tantas satisfacciones al idioma español utilizado en la República Argentina con todo el "brillo y esplendor" exigidos, y además, porque aunque lo adaptemos al sabor de nuestra tierra, no dejará de ser, ante todo, español. (Medina Verna, 1953: 19)
}

La Academia Nacional de la Lengua, que tendrá a su cargo la confección del Diccionario Nacional, terminará de una vez por todas con la prisión literaria del escritor argentino, dentro de los cánones absolutos y convencionales del idioma español, ya que podrá usar, sin temor a caer en informalidades académicas o gramaticales, los recursos de expresión que son originarios de nuestras regiones patrias, como así de toda la América latina. Y entendamos que se trata de la configuración de la lengua, y no de su desfiguración. Las academias se respetan entre sí. Son instituciones, y las instituciones de la cultura son las que hacen avanzar la humanidad. (Medina Verna, 1953: 19)

Con tales tensiones es preciso comprender la coyuntura 1952-1953: por un lado, la integración de trazos del discurso soberanista en el Estado y en publicaciones y ámbitos de auditorio amplio condiciona también aquello que puede ser enunciado; por el otro, se trata de contradicciones que estructuran el primer peronismo en tanto alianza de sectores heterogéneos, entre momentos de radicalidad en la emergencia de lo popular y momentos de preservación de las instituciones republicanas. Los discursos sobre la lengua de este período contienen, pues, huellas de tales contradicciones y heterogeneidades.

\section{Palabras finales}

Doble es el efecto que la relación centro-periferia genera en el análisis de las series presentadas. En primer lugar, aparece en un orden vinculado a la problemática que estructura el trabajo: los discursos que articulan lengua y soberanía. En este sentido, centro y periferia remiten a posiciones en un campo de relaciones internacionales de dominación, que resultan constitutivas del modo de formación de la Argentina como país políticamente autónomo. Esto es, se trata de una contradicción que no responde exclusivamente a ciertos contextos, voluntades o posiciones: es fundante del lazo objetivo y subjetivo de la ciudadanía con la lengua en estos países surgidos de procesos de conquista, colonización y emancipación.

En segundo lugar, la relación desigual centro-periferiapermite comprender aspectos de la producción y circulación de los materiales analizados. En esta segunda dirección, si bien no compartimos la concepción sistémica de Angenot (2010), su reflexión sobre las relaciones centro-periferian un estado de discurso visto en perspectiva sincrónica resultan iluminadoras para reflexionar sobre algunos aspectos de las series presentadas:

La periferia del sistema discursivo está ocupada por toda clase de grupúsculos que oponen a los valores y a las ideas dominantes sus ciencias, historiosofía, su hermenéutica social e incluso (al menos de manera embrionaria) su estética (...). Las disidencias grupusculares se saben en lucha contra el imperio de la hegemonía y en la necesidad de instalar, para mantenerse, una convivialidad a toda prueba, un encierro en su propia lógica, produciendo a la vez un discurso autosuficiente e impermeable a las influencias externas (2010: 145).

Angenot remite con esta caracterización a grupos contrahegmónicos que buscan introducir transformaciones en las formas (lingüísticas, discursivas, científicas, estéticas) y/o las relaciones sociales. LC, en este sentido, puede y no ser caracterizada como periférica Lo es por sus modos materiales de producción, por la posición desde la cual elabora y edita sus publicaciones, por el lugar -subalterno respecto de los centros académicos, políticos e intelectuales- desde el cual se autoriza para hablar y debatir sobre la lengua como campo específico de saber. Ahora bien, por otro lado, sus textos están habitados por un conjunto de lugares comunes que bien podrían considerarse nodos del entramado hegemónico de la vindicación de una "cultura nacional". Tiene, en esta línea, un sentido tradicionalista conservador, retardatario en sus formas e imaginarios: en momentos de impulso al desarrollo en Argentina del sistema de rutas y autopistas, tras la 
creación en 1932 de la Dirección Nacional de Vialidad, esta publicación se autodenomina precisamente "La Carreta”. Así, al mismo tiempo que ocupa esta posición periférica en cuanto al orden de las instituciones centrales, también acarrea, en sus tonos, un modo "viejo" de plantear las cosas: a comienzos de la década de 1940 se reiteran sin mucha innovación enunciados, tópicos, formas de decir que ya estaban presentes como hemos mostrado en la sección II- ya en la década de 1920. En la coyuntura 1940-1941 las secuencias de LC parecieran, pues, una rémora, la enunciación de algo no resuelto que regresa bajo la forma de cierta previsibilidad, enunciación polémica pero falta de fuerzas para confrontar con las figuras centrales del quehacer pedagógico prescriptivo y académico, sin capacidad de sorprender y dislocar el sentido común: hacia el pasado mira en esa cruzada contrahispanista.

La serie de LP, como nodo de una red amplia y abigarrada de textos publicados en 1952-1953, se muestra a primera vista como la realización, triunfante, de la campaña emprendida por Rossi en los años '20, por Pinto en LC y, mucho antes, por los escritos decimonónicos que auguraban la emancipación también en el gobierno de la lengua: la concreción de aquello que, diez años antes, parecía una predicación inconducente de un sector marginal. ¿Qué conduce de un escenario al otro? Una posible hipótesis es que el discurso de valoración de la soberanía y del pueblo como instancias legítimas cobra vigor al calor de las movilizaciones populares, en especial, aunque sin linealidad ni inmediatez, tras la irrupción del acontecimiento que significó para los sectores populares la emergencia del peronismo.

Con todo, es preciso destacar que en ambos casos se trata de un discurso que, con fuerte impronta política, adquiere los funcionamientos del género epidíctico: homenaje o diatriba, antes que una intervención efectiva sobre la lengua y sobre los instrumentos lingüísticos -la confección material, por caso, de diccionarios- la reivindicación de soberanía frente a las tradiciones hispanistas pareciera consistir en una declamación que, al no ponerse en práctica bajo otras formas de la materialidad, carga con la necesidad de su insistente retorno.

\section{Bibliografía}

Alfón, F. (2013). La querella de la lengua en la Argentina. Antología Buenos Aires: Biblioteca Nacional.

Angenot, M. (2010). Interdiscursividades. De hegemonías y disidenciaßórdoba: Universidad Nacional de Córdoba.

Arnoux, E. (2008). Los discursossobrela nación y el lenguaje en la formacióndel Estado (Chile, 1842-1862). Estudio glotopolíticoBuenos Aires: Santiago Arcos.

Authier-Revuz, J. (1984). Hétérogénéité(s) énonciative(s), Langages, № 73, 98-111.

Authier-Revuz, J. (1998). Palavras incertas. As não-coincidências do dizerCampinas, SP: Editora da UNICAMP.

Bentivegna, D. (2011). El poder de la letra. Literatura y domesticación en la ArgentinaLa Plata: UNIPE.

Bentivegna, D. (2018). La eficacia literaria. Configuracionesdiscursivas de literatura nacional en manuales argentinos (1866-1947). Buenos Aires: EUDEBA.

Bernecker, W. (2000). El fin de siglo en el Río de la Plata: intereses internacionales y reacciones latinoamericanas. En O. Ette y T. Heydenreich (orgs.). JoséEnrique Rodóy su tiempo.Cien años del Ariel. Frankfurt/Madrid: Verbuert/ Iberoamericana, pp. 15-39.

Bertoni, L. A. (2001). Patriotas, cosmopolitasy nacionalistas. La construcciónde la nacionalidad argentina a fines del siglo xix. Buenos Aires: Fondo de Cultura Económica.

Casas, M. E. (2016). Los lazos de los gauchos. La sociabilidad como herramienta para la difusión de la tradición en la provincia de Buenos Aires, 1930-1950, Avances del Cesorvol. 13 n 14, Rosario.

Courtine, J-J. (1981). Quelques problèmes théoriques et méthodologiques en analyse du discours, à propos du discours communiste adressé aux chrétiens, Langages, 62, 9-128.

Degiovanni, F. y Toscano y García, G. (2010). Disputas de origen: Américo Castro y la institucionalización de la filología en Argentina. Nueva Revista de Filología HispánicaT. 58 n 1, 191-213.

di Tullio, Á. (2003). Políticas lingüísticas e inmigración. El caso argentinoBuenos Aires: EUDEBA. 
Ennis, J. A. (2008). Decir la lengua. Debates ideológico-lingüísticoen la Argentina desde 1837. Frankfurt am Main, Berlin, Bern, Bruxelles, New York, Oxford, Wien: Peter Lang.

Ferrero de Sahab, G. et al. (1993). Historia de la editorial Losada, "voz" de los exiliados españoles. En L. Martínez Cuitiño, y É. Lois (coords.), Actas del III Congresode Hispanistas "España en América y América en España”. Buenos Aires: Universidad de Buenos Aires, 521-544.

Funes, P. (2006). Salvar la nación. Intelectuales, cultura y política en los años veinte latinoamericanos. Buenos Aires: Prometeo Libros.

García-Romeu, J. (2012). Rupturas en las visiones de América: de la utopía a la ucronía. Babel. Littératures plurielles $\mathrm{n}^{\circ} 26$.

Glozman, M. (2015). Lengua y peronismo. Políticas y saberes lingüísticos en la Argentina, 1943-1956. Archivo documental Buenos Aires: Biblioteca Nacional.

Glozman, M. (2017). Debates sobre la lengua, divulgación gramatical y políticas de Estado en el primer peronismo, Revisa Afuera. Estudios de crítica culturaln ${ }^{\circ}$ 17/18, ISSN: 1850-6267.

Glozman, M. (2018). Combates desde los márgenes. Luis C. Pinto en la historia de los debates y saberes sobre la lengua (Argentina, 1940-1956). En R. Bein, J. E. Bonnin, M. di Stefano, D. Lauria y M. C. Pereira (eds.), Homenajea Elvira Arnoux. Estudiosde análisis del discurso,glotopolíticay pedagogíade la lectura y la escritura. Buenos Aires: Editorial de la Facultad de Filosofía y Letras de la Universidad de Buenos Aires (en prensa).

Glozman, M. y Lauria, D. (2012). Vocesy ecos. Una antología de los debates sobre la lengua nacional (Argentina, 1900-2000). Buenos Aires: Cabiria/Biblioteca Nacional.

Guilhaumou, J. , Maldidier D. y Robin, R. (2016). Efeitos do arquivo, Discursoe arquivo. Experimentaçõesem análise do discurso.Campinas, SP: Editora da Unicamp, pp. 115-140.

Korn, G. (2017). Hijos del Pueblo. Intelectualesperonistas: de la Internacional a la Marcha. Buenos Aires: Editorial Las Cuarenta.

Lagos, G. (2014). El nacionalismo de Ricardo Rojas en tiempos del centenario (1900-1916), Cuadernosde la Facultad de Humanidades y Ciencias Sociales (Universidad Nacional de Jujuyh ${ }^{\circ} 45$.

Leonardi, Y. (coord.) (2017). Dossier “Cultura y peronismo”, Revista Afuera. Estudiosde Crítica Cultural, N 17-18. Recuperado de: http://revistaafuera17-18.blogspot.com/p/blog-page.html

Martínez Gramuglia, P. (2006). Ricardo Rojas: una modernidad argentina, Anuario del Centrode EstudiosHistóricos "Prof. Carlos S. A. Segreti,"vol. 6, n 16, 313-354.

Morgenfeld, L. (2011). Vecinos en conflicto. Argentina y Estados Unidos en las Conferencias Panamericanas (1880-1955). Buenos Aires: Peña Lillo/ Ediciones Continente.

Pêcheux, M. (2012). Análise de Discurso. Textos escolhidos por Eni Puccinelli Orlanđiampinas SP: Pontes.

Pêcheux, M. (2016). Las verdades evidentes. Lingüística, semántica, filosofíauenos Aires: Ediciones del CCC.

Rein, R. (2003). Entre el abismo y la salvación. El pacto Franco-PerónBuenos Aires: Lumière.

Rein, R. y Panella, C. (comps.) (2013). Cultura para todos.El suplementocultural de La Prensa cegetista(1951-1955). Buenos Aires: Biblioteca Nacional.

Schwarzstein, D. (2001). Entre Franco y Perón. Memoria e identidad del exilio republicano español en Argentina. Barcelona: Crítica.

Sepúlveda, I. (2005). El sueñodela Madre Patria. Hispanoamericanismoy nacionalismo.Madrid: Fundación Carolina, Centro de Estudios Hispánicos e Iberoamericanos/Marcial Pons Historia.

Terán, O. (1993). El dispositivo hispanista, Actas del III CongresoArgentino de Hispanistas "España en América y América en España". Buenos Aires, 19 al 23 de mayo de 1992, pp. 129-137.

Toscano y García, G. (2013). Language debates and the institutionalization of philology in Argentina in the first half of the twentieth century. En J. del Valle (ed.), A political historyofSpanish. The Making ofa Language, Cambridge, Cambridge University Press, pp. 212-228.

Varela, L. (1999). Ideas sobre el lenguaje y proyectos de país. Posiciones en el debate de 1837. En E. Arnoux y R. Bein (comps.), Prácticas y representaciones del lenguajBuenos Aires: EUDEBA, pp. 17-35. 


\section{Materiales}

Arlt, R. (1958) [1930]. El idioma de los argentinos, Aguafuertes Porteñas Buenos Aires: Losada.

Cambours Ocampo, A. (1952). El problema de nuestro lenguaje. La Prensa, 3/2/1953.

Medina Verna, A. (1953). Independencia de nuestro idioma nacional. La Prensa, 25/10/1953.

Perón, J. D. (1947). Discurso de S. E. el Señor Presidente de la Nación General Juan D. Perón en la sesión de homenaje a Cervantes, Boletín de la Academia Argentina de LetrasXVI, pp. 473-500.

Pinto, L. C. (1940a). Amado Alonso y 'su Idiomilla de Coleccionista'. La Carreta, nº 97, 5-8.

Pinto, L. C. (1940b). ¿Hispanofobia?. La Carreta, n 98, 19-20.

Pinto, L. C. (1940c). Señor gramatiquero. La Carreta, n 100, 5-7.

Pinto, L. C. (1941a). Por nuestro idioma nacional. La Carreta, n 103, 3-5.

Pinto, L. C. (1941b). Sueño de 'Imperio Idiomático'. La Carreta, n’ 108, 3.

Pinto, L. C. (1941c). Américo Castro 'Corregidor de la Lengua'. La Carreta, no 111, 3-5.

Quesada, E. (1899). El problema de la lengua en la América española, Revista Nacional, tomo XXVIII, 241-257.

Rossi, V. (1926). Cosas de negros.Córdoba: Imprenta argentina.

Rossi, V. (1929). Idioma nacional rioplatense.Cuarta evidencia. FolletosLenguaraces, No 9. Río de la Plata: Casa Editora Imprenta Argentina.

Rossi, V. (1941). A los Encomenderos Idiomáticos de los Pueblos del Plata. La Carreta, nº 111, 7.

Sarmiento, D. F. (1843). Memoria (sobreortografía), leída a la Facultad de Humanidades. Santiago de Chile: Imprenta de la Opinión. 